\title{
On the free radical bulk polymerization of styrene in the presence of recycled rubber particles: a kinetic study using DSC
}

\author{
Daniela Florez, Sandrine Hoppe, Guo-Hua Hu, Dimitrios Meimaroglou
}

August 24, 2018

\begin{abstract}
The kinetic developments of the free-radical bulk polymerization of styrene in the presence of recycled rubber tire particles are studied by isothermal differential scanning calorimetry (DSC). Two different chemical initiators, namely benzoyl peroxide (BPO) and 2,2'-Azobis(2methylbutyronitrile) (AMBN), are tested in a series of variable-composition experiments, under three different polymerization temperatures, in order to selectively address the role of the different species on the evolution of the polymerization reaction. The variation of the overall effective kinetic rate constant and activation energy of the polymerization are also calculated to quantify the effect of the addition of the particulate filler. The results of the study demonstrate that the combination of the peroxide initiator agent with a composition of the reacting mixture in the order of 30 mass\%, in terms of the rubber tire particles, results in a significant deviation of the evolution of the rate of polymerization and monomer conversion, with respect to that of the pure homopolymerization system in the absence of tire particles. The observed phenomena are consistent with previous studies reported in the literature and are mainly attributed to the chemical interactions taking place between the initiator agent and additives of the formulation of the tire particles.
\end{abstract}

Keywords DSC, polymerization kinetics, styrene, ground rubber particles, tire recycling.

\section{Introduction}

The over-accumulation of used tires has become, over the last decades, a significant ecological issue drawing the attention of scientists on a worldwide scale. Among the different routes of treatment of end-of-life tires (e.g., land-filling, energy recovery, reuse, etc.), the recycling of these tires appears as a sustainable solution offering significant benefits related to its low environmental impact and important production of secondary raw materials with high economic value [1, 2]. In this context, one method for tires' recycling consists in the mechanical grinding of the rubber-containing parts to fabricate micrometric powders, commercially called ground tire rubber (GTR). These particles are constituted of a three-dimensional network of natural and synthetic cross-linked elastomer chains, reinforcing agents, curatives and additives [3]. It is exactly because of its elastomeric properties that GTR has found widespread applications as filler agent in the formulation of polymer composites $[4,5]$.

Polystyrene (PS) is a thermoplastic commodity, widely used in several consumer products, whose mechanical properties (i.e., notably related to its hardness and stiffness) can be modified by the addition of rubber into the polymer matrix, thus producing a polystyrene grade known as high-impact polystyrene [6]. Hence, several research studies have been focused on the idea of replacing fresh rubber with GTR particles in order to achieve a similar improvement on the mechanical properties of PS and to recycle used tires at the same time [4]. The main issue with this concept is that the two phases (i.e., the GTR particles and polystyrene matrix) display low adhesion towards one another, making it difficult to produce an homogeneous compound simply by blending them. A commonly adopted strategy to overcome this obstacle and render the two phases compatible is to graft the polymer directly onto the surface of the GTR particles via in-situ free-radical polymerization.[6-8]. 
The presence of particulate fillers in a free-radical polymerization system may often alter, directly or indirectly, the mechanism of the reaction [9-11]. In fact, depending on their physicochemical characteristics, the fillers can interact with other reactive species present in the mixture, in a series of parallel and/or competitive chemical reactions, resulting in the modification of the expected evolution of the system in terms of the measured conversion, yield and product properties. As a result, the polymerization can be either accelerated or severely retarded and/or inhibited solely by the presence of an additive that displays strong interactions with the initiator agent(s) and/or the monomer(s). This seems to be also the case for free-radical polymerization systems in the presence of GTR since, depending on the nature of the initiator agent, the type of the monomer and the amount and composition of GTR, an important acceleration or inhibition can be observed on the polymerization reaction. For example, Coai and coworkers [8] have observed a significant decrease in the final monomer conversion of styrene, when using bezoyl-peroxide (BPO) as initiator agent, contrary to organic azo-initiators such as azobis(isobutyronitrile) (AIBN) that displayed no significant effect. In contrast, Xiong [12] demonstrated that in the presence of scrap tire rubber powder, the overall polymerization rate of acrylate monomers such as methyl methacrylate (MMA), glycidyl methacrylate (GMA) and hydroxy ethyl methacrylate (HEMA) is accelerated, if the reaction is initiated with BPO. In these studies, the observed effects were attributed to the interactions between the initiators, the monomers and several additives present in the GTR formulation, such as carbon black, the most widespread rubber tire reinforcing agent [3].

The main purpose of this study is to investigate the effects of the presence of GTR particles on the free-radical polymerization kinetics of styrene by using differential scanning calorimetry (DSC). DSC is a sensitive and precise thermal analysis technique that has proven to be a useful tool in the measuring of polymerization kinetics $[9-11,13]$. In this technique, the heat flow, which is assumed to be proportional to the reaction rate evolution, is recorded during the course of the reaction. In the case of the polymerization of vinyl monomers, variations in the polymerization rate exhibit a significant exothermic heat release. Although some research has been carried out on the preparation and characterization of composites based on PS and GTR [4, 6, 8], to the best of the authors' knowledge, it is the first time that a systematic study of the kinetics of the bulk free-radical polymerization of styrene in the presence of GTR particles is carried out using DSC, under different conditions of temperature and composition.

\section{Experimental}

\subsection{Materials and reagents}

All the reagents used, namely, styrene monomer (with a purity $\geq 99.5 \%$ and stabilized with $\sim 0.005 \%$ of 4-tert-butylcatechol), 2,2'-Azobis(2-methylbutyronitrile) AMBN (with a purity $\geq 98$ $\%$ ) and BPO (75\% remainder water), were purchased from Sigma-Aldrich and used without further purification. Commercial GTR was obtained from DeltaGom France and was used without purification, unless otherwise stated. This specific grade of GTR was derived from the recycling of end-of-life tires of different categories (light, heavy goods and agricultural vehicles, motorcycles, etc.) and is mainly constituted of a blend of styrene-butadiene rubber and natural rubber, and several additives (such as aromatic oils, organic chemicals, vulcanizing and reinforcing agents). It came in the form of powder with a mean particle size in the order of $635 \mu \mathrm{m}$. The purification of GTR particles, for some measurements, was carried out by extraction with chloroform during 48 hours, under permanent stirring, followed by vacuum drying until constant weight.

\subsection{Methods}

The GTR particles used in this study are able to absorb more than twice their weight in styrene. To ensure homogeneous repartition of styrene around and within the GTR particles, particular attention was paid to the preparation of the reaction medium. Hence, before each measurement, the monomer solution was initially prepared by dissolving the desired amount of initiator agent in styrene under constant stirring during 15 minutes. In parallel, the required amount of GTR particles, according to the desired composition, was uniformly deposited, in the form of a thin layer, on the internal surface of a cylindrical shaped glass container. The monomer solution was 
subsequently added drop by drop, for samples containing $30 \mathrm{wt} \%$ of GTR, or sprayed over the particles, for samples with $50 \mathrm{wt} \%$ of GTR. Finally, the glass flask was sealed and stored at $4{ }^{\circ} \mathrm{C}$ for 6 hours before measurement.

All DSC thermograms of the styrene free-radical bulk polymerization, in the presence and absence of GTR, were recorded using a DSC device from Texas Instruments, model Q2000. The samples were placed into hermetic aluminum pans, specially adapted for volatile products, and scanned in two consecutive runs. The isothermal reactions were conducted during the first scan; subsequently, the samples were cooled down to $50{ }^{\circ} \mathrm{C}$ before a second, non-isothermal scan at 5 ${ }^{\circ} \mathrm{C} \min ^{-1}$ from $50{ }^{\circ} \mathrm{C}$ to $220{ }^{\circ} \mathrm{C}$, to assure complete monomer conversion. During the scans, the sample temperature $\left( \pm 0.01{ }^{\circ} \mathrm{C}\right)$ and the heat flow $\left(\mathrm{W} \mathrm{g}^{-1}\right)$ were recorded as a function of time. Before any further treatment and exploitation of the results, the recorded heat flow data were normalized with respect to the mass of styrene, $\left(\mathrm{W} g_{s t y}^{-1}\right)$. Finally, the total sample mass was verified before and after each measurement. Eventual losses in mass, recorded for certain samples, were always inferior to $2 \%$.

On the basis of the recorded curves, the time-evolution of monomer conversion can be directly determined from the heat flow rate by the following expression [10, 13]:

$$
\frac{d x}{d t}=\frac{1}{\Delta H_{T}} \frac{d(\Delta H)}{d t}
$$

where $d(\Delta H)$ is the instantaneous reaction enthalpy, assigned to the corresponding area under the DSC curve, and $\Delta H_{T}$ is the total enthalpy defined as the sum of the recorded enthalpies. Note that, when GTR was present in the samples, the volatile substances present in its formulation caused a burst-off of the lid of the pans during the second dynamic scan, resulting in greater mass losses. Hence, in these cases, only the first isothermal scan was carried out and the total reaction enthalpy, recorded for the homopolymerization samples and which was in agreement with the published literature values, was used as reference.

\section{Results and discussion}

\subsection{Monitoring of the styrene-free radical polymerization.}

At a first stage, the course of the isothermal free-radical bulk polymerization of styrene, in the absence of GTR, was monitored by DSC at three different temperatures, ranging from $85{ }^{\circ} \mathrm{C}$ to $120{ }^{\circ} \mathrm{C}$. Chemical initiation was promoted by the use of two different initiator agents, namely $\mathrm{BPO}$ and $\mathrm{AMBN}$, which were implemented separately (i.e, a single initiator agent was used in each polymerization experiment, either AMBN or BPO). The DSC thermograms shown in Figure 1a display the evolution of the heat flow with time for four different polymerizations carried out at $85{ }^{\circ} \mathrm{C}$ and $90{ }^{\circ} \mathrm{C}$ and in the presence of the two initiator agents. On these curves, one can distinguish the inflection point that follows the initial polymerization stage and marks the onset of the auto-acceleration stage; in this stage, termination reactions become diffusion-controlled and a marked increase on the rate of polymerization is observed, evident by the parallel increase in the released heat-flow. Subsequently, at very high monomer conversion, even the propagation reactions become diffusion-controlled resulting in a decrease on the rate of polymerization until complete monomer conversion [10, 13-15]. The dynamic evolution of monomer conversion, calculated according to Equation (1), is plotted in Figure 1b. The existence of an autoacceleration stage can be easily identified by the characteristic "S" shape of the curves. As expected, both AMBNinitiated polymerizations proceed at higher reaction rates with respect to the BPO-initiated ones at the same temperatures, due to the significantly lower half-life time of AMBN at both temperatures.

The total reaction enthalpies, calculated by numerical integration of the thermogram data, were found to vary in the range between 74 and $79 \mathrm{~kJ} / \mathrm{mol}$ for all polymerizations in the absence of GTR. These values are in agreement with the literature values on the heat of styrene polymerization, [16], thus verifying complete monomer conversion in all cases. 


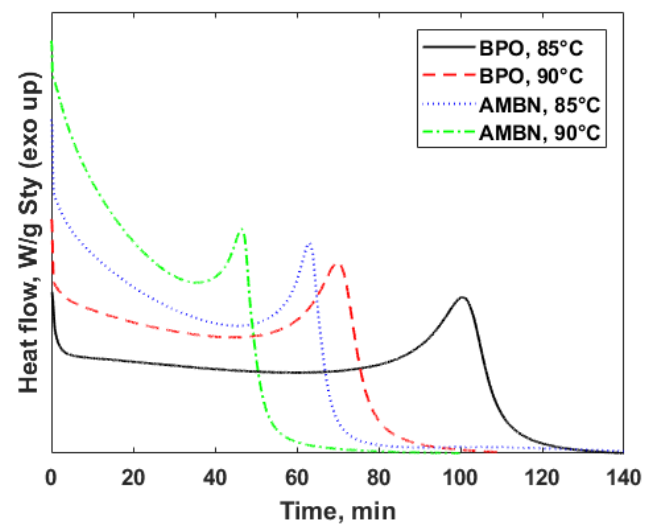

(a)

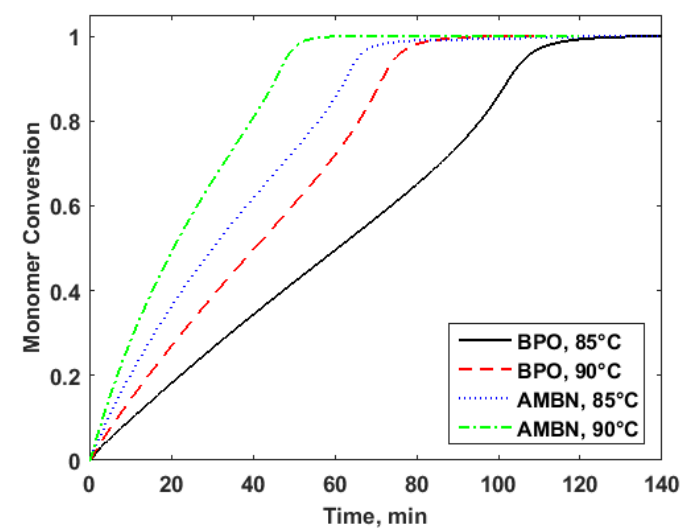

(b)

Figure 1: Effect of the initiator type and temperature on a) heat flow and b) monomer conversion in function of time during isothermal free radical bulk polymerization of styrene by using a ratio initiator to styrene of $4.6 \% \mathrm{wt}$.

\subsection{Monitoring of the styrene-free radical polymerization in the pres- ence of GTR.}

GTR is a polymer network of elastomers reinforced with carbon black particles and several other additives [1,5]. As mentioned earlier, several studies have elucidated different phenomena that take place in free-radical polymerization systems containing GTR particles. Among the phenomena studied, the effects of carbon black possess a dominant position as its presence may severely affect the chemistry of the system [17-19]. These effects can be more or less pronounced, according to the chemical structure of the carbon black surface and to the nature of monomers and initiator agents. At the same time, the cross-linked nature of the GTR as well as the different additives and impurities, present in the GTR formulations, may also display a significant effect on the course of free-radical polymerizations. These latter phenomena are present in the literature in a less systematic way due to the uncertainties related to the exact identification and quantification of the role of the different additives of GTR [12]. Hence, the behavior of such systems often displays a deviation from the commonly expected evolution of the pure free-radical polymerization systems (i.e., without the presence of GTR particles) that, in certain cases might be very pronounced, depending on the amount and the composition of the GTR as well as on the nature of the monomer(s) and initiator agent(s). In this respect, in an attempt to identify some of the aforementioned effects, a series of DSC thermograms has been produced for the free-radical polymerization of styrene in the presence of GTR particles, under three different temperatures (i.e, $85{ }^{\circ} \mathrm{C}, 90{ }^{\circ} \mathrm{C}$ and 120 ${ }^{\circ} \mathrm{C}$ ) and for three different concentrations of GTR in the system (i.e., 0\%, 30\% and 50\% wt). At the same time, two different initiator agents were used, namely BPO and AMBN, while their concentration was also varied. The conditions of the different experiments are presented in Table 1.

Table 1: Composition of the different polymerizations monitored. All conditions were performed for both $\mathrm{BPO}$ and $\mathrm{AMBN}$, varying the ratio of initiator (I) to styrene (St) at three different temperatures.

\begin{tabular}{cccc}
\hline $\begin{array}{c}\text { GTR } \\
(\text { wt\% })\end{array}$ & $\begin{array}{c}\text { Initiator } \\
(\text { wt\% })\end{array}$ & $\begin{array}{c}\mathrm{I} / \mathrm{St} \\
(\mathrm{wt} / \mathrm{wt} \%)\end{array}$ & $\begin{array}{c}\mathrm{I} /(\mathrm{St}+\mathrm{GTR}) \\
(\mathrm{wt} / \mathrm{wt} \%)\end{array}$ \\
\hline 0 & 4.6 & 4.6 & \\
50 & 2.3 & 4.6 & 2.3 \\
30 & 3.1 & 4.6 & 3.2 \\
30 & 4.5 & 6.6 & 4.7 \\
50 & 4.5 & 9.4 & 4.7 \\
\hline
\end{tabular}




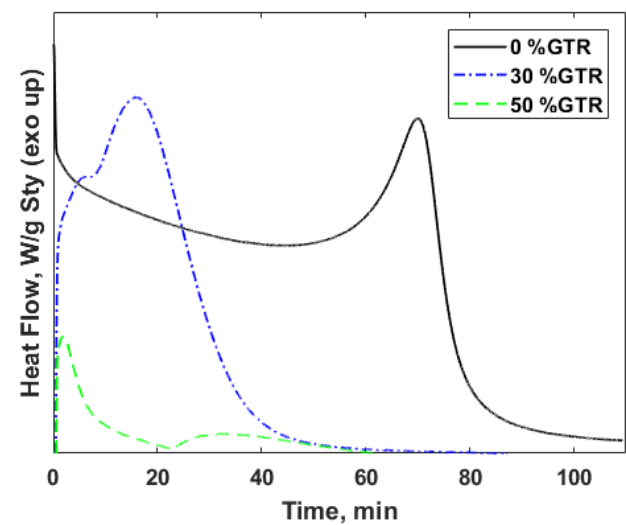

(a)

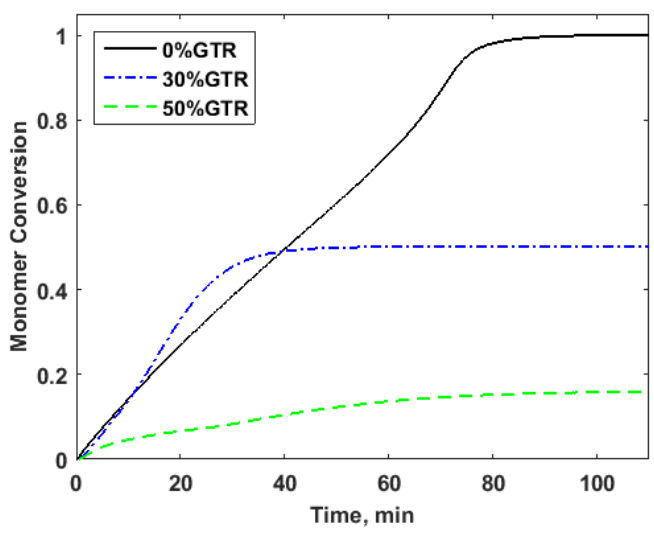

(b)

Figure 2: Effect of the GTR content on the evolution of a) heat flow and b) monomer conversion, during the isothermal BPO-initiated polymerization of styrene in the presence of GTR particles. $\left(\mathrm{T}=90{ }^{\circ} \mathrm{C}\right.$; ratio $\left.\mathrm{BPO} / \mathrm{St}=4.6 \mathrm{wt} \%\right)$.

\subsubsection{Effect of the GTR content}

An initial assessment of the primary effect of the presence of GTR in the free-radical bulk polymerization of styrene can be made by comparing the evolution of monomer conversion, produced by the corresponding DSC thermograms, in the presence of the three different amounts of GTR and at constant temperature and initiator content (i.e., with respect to the amount of styrene), as shown in Figure 2. It can be seen that the addition of GTR clearly affects both the initial rate of polymerization and the overall monomer conversion. More specifically, an increase in the GTR content shifts the exothermic peak of the maximum polymerization rate to the left (i.e., towards lower reaction time), as mostly evident by the heat flow curves shown in Figure 2a. At the same time, the overall monomer conversion is reduced with the increase of the GTR content (cf. Fig.2b).

The present findings seem to be consistent with those of similar studies reported in the literature. Coiai et al., 2006 [8], found that the BPO-initiated polymerization of styrene in the presence of $10 \mathrm{wt} \%$ GTR did not exceed $54 \%$ in monomer conversion, even after 24 hours at $85{ }^{\circ} \mathrm{C}$, in contrast to the homopolymerization case (i.e., in the absence of GTR) where the final conversion reached $99.6 \%$ under the same conditions. They also observed another almost two-fold decrease in the final monomer conversion when increasing the GTR content up to $30 \mathrm{wt} \%$. In a different study [17], it was found that carbon black significantly increased the decomposition rate of peroxide initiators such as BPO [19]. Finally, numerous studies have demonstrated that the peroxide-initiated polymerization of vinyl monomers is catalyzed, inhibited and/or retarded in the presence of carbon black $[12,17-20]$.

In fact, the surface of carbon black is mainly covered with oxygen-containing groups, such as carboxyl-carbonate structures, phenolic or alcoholic hydroxyl groups and carbonyl groups, isolated or present in quinone groups. At the same time, some of the carbon atoms have unsatisfied valencies, leading to a high electron transfer reactivity [21]. As a result, when the polymerization is initiated by an organic peroxide, such as BPO, the dissociated benzoyl acid radicals may react with the unpaired electrons to give rise to new surface-active sites, to which primary and growing polymer radicals compete to react. The reactivity of the newly formed radicals is not completely inert and can therefore undergo further reactions mainly with propagating chains. On the other hand, it is well known that the combination of peroxides with reducing agents is common in producing radicals at reasonable rates over a wide temperature range [15]. Therefore, when BPO gets in contact with the numerous oxide groups on the surface of carbon black, it suffers a strong redox reaction [19]. The redox decomposition reaction is favored at relatively low temperatures due to its activation energy (i.e., $40-60 \mathrm{~kJ} / \mathrm{mol}$ ) that is significantly lower than that of the thermal decomposition one (i.e., $120-150 \mathrm{~kJ} / \mathrm{mol}$ ) [12, 15]. 
From the above it becomes apparent that carbon black, present in the composition of GTR, can produce various and sometimes even antagonistic effects within the polymerization system $[17,18,20,22,23]$. Besides its catalytic effect on the decomposition of peroxide agents, it can also display an inhibitive or promoting effect on the polymerization, depending on the nature and the polarity of the participating monomer(s). Hence, peroxide-initiated polymerizations of vinyl monomers with negative e-value ${ }^{1}$ [24], are commonly inhibited in the presence of GTR, whilst peroxide-initiated polymerizations of positive e-valued monomers are rather favored in the presence of GTR. For example, Xiong (2010) reported that in the BPO initiated-polymerization of acrylate monomers such as MMA, in the presence of $4 \mathrm{wt} \%$ of GTR and $0.375 \mathrm{~mol} \%$ of BPO at only $50{ }^{\circ} \mathrm{C}$, the reaction was significantly accelerated and the conversion was almost eight times higher than those of the pure monomer (i.e. in absence of GTR). In contrast, when replacing the GTR by the same rubber tire formulation (elastomer, aromatic oils, curatives and additives) but without carbon black, the reaction was moderately retarded in regard to the neat monomer at the same conditions.

It is exactly the combination of the above phenomena that can explain the effects of the addition of GTR in the bulk free-radical polymerization of styrene, as observed in Figure 2. The acceleration observed with the addition of GTR can be attributed to the catalytic effect of carbon black on the decomposition of BPO, increasing the generation of primary radicals from the early stages of polymerization. Ideally, the produced primary radicals should react either with monomer molecules to initiate polymer radicals, either with the unsaturated bonds at the surface of GTR (i.e., that exist within the polymer chains of the elastomers of GTR) and subsequently with monomer molecules in order to initiate grafted polymer radicals. Nonetheless, a third class of reactions, parallel and antagonistic to the previous ones, seems to contain the aforementioned reactions of BPO (and primary radicals) with the surface of carbon black, leading to low reactive radicals and non-reactive species. It is this latter class of chemical reactions that may be responsible for the significant inhibition of the polymerization and the overall decrease of the final monomer conversion.

Finally, another reason that could partially contribute to the initial acceleration of the reaction rate is the morphological three-dimensional structure of the rubber particles. Similar to the wellknown auto-acceleration or gel-effect (or Trommsdorff effect) [25], commonly observed in certain bulk free-radical polymerization systems, the cross-linked structure of the elastomer network of GTR could also limit the mobility of long radicals, thus producing a decrease of their apparent termination rate. To prove this, Xiong [12] increased the viscosity of the reacting media by dissolving a certain amount of poly(methyl methacrylate) (PMMA) into the initial MMA-BPO solution. His findings confirmed that the polymerization rate was accelerated from the start to the end and therefore, the course of the reaction exhibited a similar behavior to that observed in presence of rubber particles.

\subsubsection{Effect of the initiator nature}

In order to further investigate and eventually confirm the above effects of BPO, a set of DSC thermograms has been produced under the same conditions, but using a different initiator agent, namely AMBN. The corresponding monomer conversion evolution curves are shown in Figure 3. As can be seen, the effect of the amount of GTR on the evolution of the polymerization is markedly less pronounced; in fact, the previous acceleration effect seems to have disappeared while the inhibition effect is severely diminished, with the final monomer conversion reaching $100 \%$ in both $0 \%$ and $30 \%$ GTR content, while being reduced only to about $91 \%$ at $50 \%$ GTR content. At the same time, the presence of GTR seems to display a retardation effect on the evolution of the polymerization.

Unlike peroxides that, as mentioned above, are highly reactive with the surface of carbon black resulting in pronounced effects on the evolution of peroxide-initiated polymerization systems, azocompounds are less active towards the surface of carbon black. Hence, regardless of the monomer e-value, polymerizations initiated by azo-compounds are only moderately retarded in the presence

\footnotetext{
1 According to the Alfred and Price's Q and e scheme, the e-value is a constant characteristic value for each individual vinyl monomers that takes into account for the polarity of their double bonds; electron-rich (negative e) and electron-poor (positive e), and which influences their reactivity [24].
} 


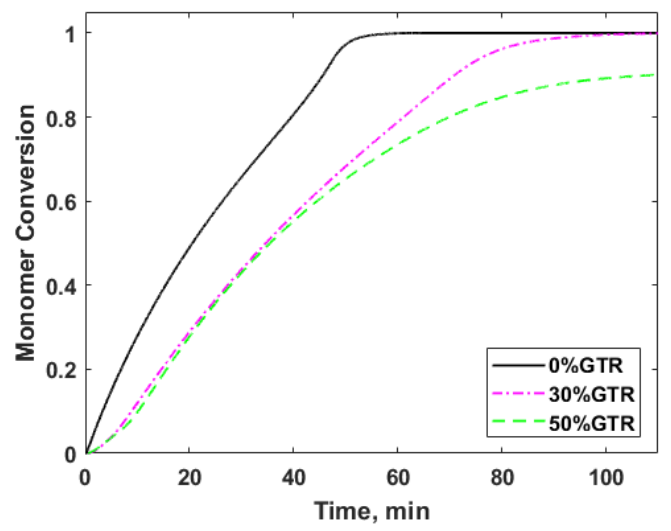

Figure 3: Effect of the GTR content on the evolution of monomer conversion during the isothermal AMBN-initiated radical polymerization of styrene, in the presence of GTR particles. $\left(\mathrm{T}=90^{\circ} \mathrm{C}\right.$; ratio $\mathrm{AMBN} / \mathrm{St}=4.6 \mathrm{wt} \%)$.

of GTR [20, 26]. This difference in the behavior and in the effects of the two types of initiator agents has been reported in numerous studies in the literature. Ohkita et al., (1975) [18] observed that the BPO initiated radical polymerization of styrene was inhibited in presence of furnace blacks. In this study, the overall monomer conversion was limited to only $2 \%$ when adding 2 wt $\%$ of carbon black $\left(1.5 \cdot 10^{-4} \mathrm{~mol} \%\right.$ of BPO), instead of $18 \%$ in the absence of carbon black after 10 hours of reaction. Inversely, when AIBN was used as initiator agent, only a moderate retardation was observed on the evolution of monomer conversion under the presence of carbon black of approx. $25 \%$ with respect to that of the case without carbon black. Coiai et al., 2006 [8], in contrast to their aforementioned observations regarding the inhibition effect of GTR on the BPO-initiated polymerization of styrene (i.e., $54 \%$ monomer conversion after 24 hours at $85{ }^{\circ} \mathrm{C}$, in the presence of $10 \mathrm{wt} \%$ GTR), observed complete monomer conversion under the same conditions, simply by switching the initiator agent to AIBN.

\subsubsection{Effect of the GTR formulation}

The role of carbon black, existing in the formulation of GTR, is certainly important as shown previously and as reported by numerous studies [17, 20, 22, 23, 26]. Nevertheless, it is expected that other additives in GTR might also display an effect, either positive or negative, on the rate and the extent of the polymerization. For example, the antioxidant agents, added in the tires formulation, are designed to scavenge and destroy oxy radicals in order to slow-down the rubber aging process [27]. Hence, a certain interaction with the polymerization primary radicals and macroradicals is expected to take place. On the other hand, it is also expected that some of the GTR additives might also interact with the carbon black surface, thus partially "masking" its effects. Unfortunately, it is very difficult to isolate the extent of the positive or negative contributions of each component.

In the present study, the phenomena related to the formulation of GTR, in terms of the existence of additives and impurities, has been assessed by running a series of identical BPO-initialized polymerizations as the ones presented above, with the difference of having purified (i.e., by treatment with chloroform, cf. Sec. 2.2) the GTR particles before the experiments. Figure 4 presents a comparison of the monomer conversion curves produced by the respective thermograms, for the pure homopolymerization case and for the $30 \%$ and $50 \%$ treated (dashed lines) and non-treated (solid lines) GTR compositions. As can be seen, the inhibition effect seems to be more pronounced in the case of the "purified" GTR, with respect to the non-treated GTR.

This can be attributed to possible interactions of the additives and impurities of the GTR formulation (i.e., for the non-treated GTR) with the surface of carbon black, thus reducing the extent of interactions with the benzoyl radicals of BPO. When these additives are removed (i.e., for the treated GTR), a greater number of active radicals on the carbon-black surface are exposed, 


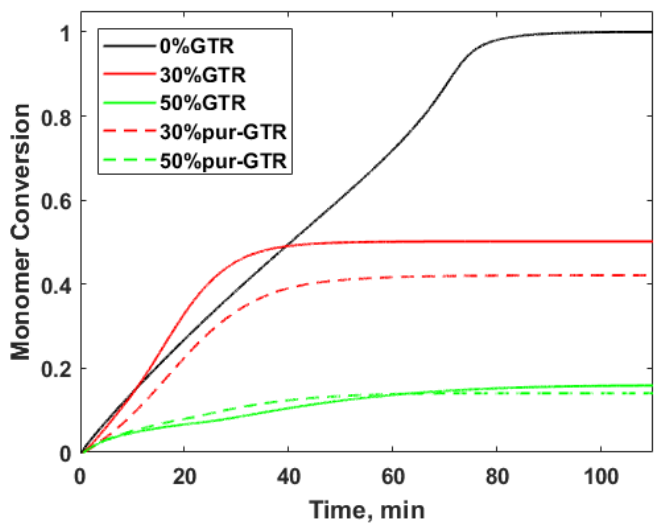

Figure 4: Styrene conversion vs time in presence of $30 \mathrm{wt} \%$ of untreated GTR and chloroformtreated GTR (pur-GTR) at $90^{\circ} \mathrm{C}(\mathrm{BPO} / \mathrm{St}=4.6 \mathrm{wt} \%)$.

able to scavenge the free benzoyl radicals and the growing polystyrene chains. Note that this does not exclude that, in the case of non-treated GTR, a part of the benzoyl radicals might be deactivated in reactions with certain additives and impurities, thus resulting in a partial inhibition of the polymerization reaction as well. But it seems that the net overall prevailing effect, when removing these additives and impurities of the GTR formulation, is an increase on the initially observed effect of GTR (i.e., in this case, a more pronounced inhibition). Similar observations have been reported in the literature. For example, Xiong (2010) [12] observed that, the promoting effect of GTR on the bulk polymerization of MMA, attributed to the interactions with the surface of carbon black, was further amplified upon purification of the rubber particles by methyl ethyl ketone. On the other hand, when removing carbon black from the GTR formulation, a significant retardation (i.e., of about $40 \%$ at $50{ }^{\circ} \mathrm{C}$ ) on the monomer conversion was observed, that could be attributed to eventual interactions of the radicals with the other additives and impurities of the GTR formulation.

\subsubsection{Effect of the initiator content and reaction temperature}

In the above Figures, the initiator content, with respect to the monomer content, and the reaction temperature have been kept constant in order to isolate their eventual influence on the rate and extent of the polymerization from the studied effects of the GTR content, the initiator agent type and the GTR formulation. To assess the effect of the initiator content, the thermograms of the polymerization have been recorded for a constant ratio of BPO over the total sample mass, in contrast to the initial measurements where the content of BPO was kept constant only with respect to the mass of styrene (c.f. Table 1). Figure 5 shows the comparison between the final monomer conversion values, achieved under the three different GTR contents in the case of constant BPO to styrene ratio (filled circles) and in the case of constant BPO to [styrene+GTR] ratio (open squares). As discussed previously, when the mass of initiator is constant with respect to the mass of styrene, a monotonous decrease is observed in the final monomer conversion. On the other hand, when the ratio of $\mathrm{BPO}$ to total sample mass is kept constant, meaning that the amount of initiator is higher in comparison to the previous case (i.e., except for the pure styrene polymerization), a rather surprising phenomenon is observed; the final monomer conversion drops very rapidly to a value around $20 \%$, even for the $30 \%$ composition in GTR, and then remains relatively invariant when increasing the GTR content to $50 \%$.

Previous studies [12] have suggested that there might exist a range of GTR content where its effects on the polymerization reactions are more pronounced, while being relatively insensitive to variations in the GTR content outside this range. The same phenomenon is observed in Figure 6, where the variation of the final monomer conversion with respect to the GTR content is depicted for a constant BPO to styrene ratio and for three different temperatures. Once again, the case of $30 \%$ GTR content seems to display the highest sensitivity towards the temperature variations, in contrast to the $50 \%$ GTR content where the monomer conversion remains rather constant with 


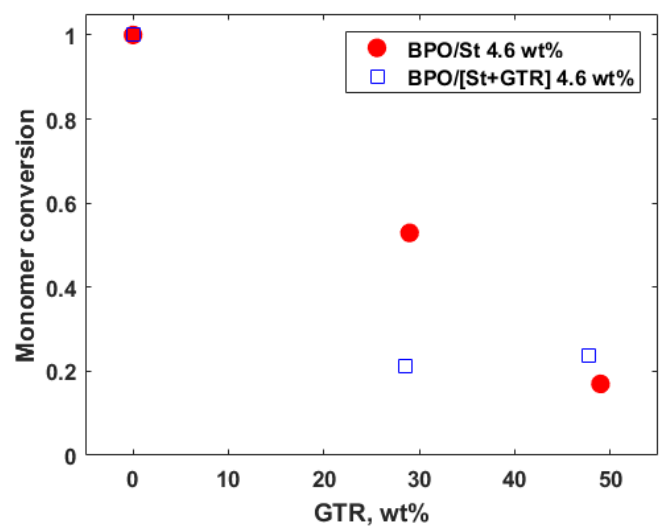

Figure 5: Influence of the GTR content on the final conversion of the BPO-initiated styrene polymerization at $90{ }^{\circ} \mathrm{Cfor}$ two different cases: $\mathrm{BPO} / \mathrm{St}$ and $\mathrm{BPO} /[\mathrm{St}+\mathrm{GTR}]$ equal to $4.6 \mathrm{wt} \%$.

respect to the temperature changes.

In both of the above cases, it is seen that an increase in either GTR content or reaction temperature brings about a more pronounced decrease on the final monomer conversion of styrene, hence a more amplified effect of GTR on the course of polymerization, as identified by the initial measurements with varying GTR content (cf. Fig. 2). Should the initial hypothesis of dominant interactions between the benzoyl radicals and the growing polymer radicals with the surface of carbon black hold true, the above observations are consistent with this hypothesis in the sense that these interactions should be more pronounced at higher GTR content or temperature. At the same time, a continuous increase in the GTR content, after a certain point, probably results in diffusional and/or steric limitations that partially compensate the above interactions, making the effects less pronounced. Since only the $30 \%$ and $50 \%$ GTR contents were tested in this study, it is not possible to better identify the range of highest sensitivity, which seems to be located between the $0 \%$ and $30 \%$ contents.

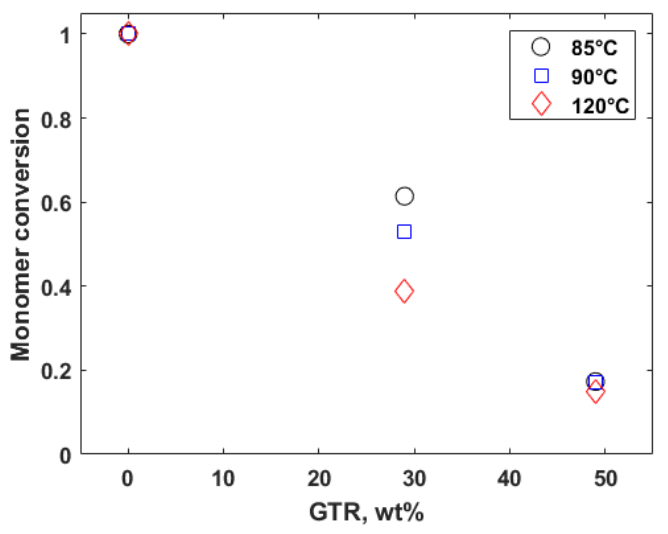

Figure 6: Influence of the temperature on the final monomer conversion of the BPO-initiated styrene polymerization in presence of GTR (BPO/St $4.6 \% \mathrm{wt})$.

\subsection{Kinetics of the polymerization of styrene in the presence of GTR.}

\section{Overall kinetic rate constants and activation energies}

As illustrated in the previous sections, the free-radical polymerization of styrene in the presence of GTR is a system that presents a complex kinetic mechanism that has not been completely elucidated. Nevertheless, in an effort to quantify the effect of GTR on the reaction kinetics, the 
overall effective kinetic rate constant and activation energy of the polymerization can be calculated on the basis of the DSC data [9, 10, 28]. Accordingly, considering an oversimplified general kinetic scheme (i.e., containing only the radical initiation, propagation and termination reactions), the polymerization rate $R_{p}$ will be given by:

$$
R_{p}=-\frac{d[M]}{d t}=R_{i}-R_{t}
$$

where $[M], R_{i}$ and $R_{t}$ denote the monomer's concentration and the radical initiation and termination rates, respectively. Note however, that the form of this expression depend on the possible existence of inhibition phenomena, in which case the rate of the inhibition reaction $R_{z}$ should be also considered (subtraction on the right side of Eq. (2)) and, in turn, the overall inhibition rate constant, $k_{z}$, needs to be determined. Unfortunately, prediction of $k_{z}$ involves difficult experimentation, since the rates to be detected are very small, in particular when the inhibition is considerably strong (e.g., BPO initiated styrene polymerization with $50 \mathrm{wt} \%$ of GTR, see figure 2b) [15]. Despite this difficulty, data from DSC measurements were treated (as described in the Appendix at the end of this paper), in an attempt to quantify the effect of the inhibitor on the overall kinetics. Nevertheless, the results obtained for the BPO-initiated reactions were not conclusive and further data collection is required to determine with confidence the constants of the inhibited polymerization.

By considering the aforementioned limitations and therefore, assuming that the effects of GTR inhibition are already implicit in the values of $k_{i}, k_{p}$ and $k_{t}$, the overall effective kinetic constants were predicted. Under the hypothesis of LCH (i.e., Long-Chain Hypothesis) and QSSA (i.e., Quasi Steady-State Assumption) for the polymer radicals [29], the differential equation above can then be written as Eq. (3) and then integrated over the initial instances of polymerization (i.e., for low monomer conversion values), by considering that the variation of the initiator concentration is negligible, to yield the expression in Eq. (4):

$$
\begin{gathered}
R_{p}=-\frac{d[M]}{d t}=k_{p}\left(\frac{f k_{d}}{k_{t}}\right)^{1 / 2}[I]^{1 / 2} \\
-\ln (1-x)=k t ; \quad k=k_{p}\left(\frac{f k_{d}}{k_{t}}\right)^{1 / 2}[I]^{1 / 2} \cong \text { const }
\end{gathered}
$$

Equation (4) can be implemented to estimate the overall kinetic constant, $k$, by plotting the linear relation $-\ln (1-x)$ versus $t$. Note that expressions in Eq. (3) and (4) are valid only under the aforementioned assumptions and their applicability should be limited to low monomer conversion values, to avoid significant variation of the value of the initiator concentration. Once the value of $k$ has been estimated for different reaction temperatures, the overall effective activation energy $E_{\text {eff }}$ of the polymerization can be inferred by considering an Arrhenius-type variation of $k$ and plotting the values of $\ln (k)$ vs $1 / T$. The above procedure was implemented to calculate the values of $E_{e f f}$, under different GTR and initiator contents. Figure 7 shows the curves obtained for the BPO-initiated styrene polymerization with $30 \mathrm{wt} \%$ of GTR while the rest of the calculated values of $E_{\text {eff }}$ are presented in Table 2.

In general, for the estimation of $k$ and $E_{e f f}$, the linear regression fitted well to the data in the ranges considered, as indicated also by the calculated values of the determination coefficient, $R^{2}$. The calculated activation energy of the homopolymerization case (i.e., in the absence of GTR) was $92.7 \mathrm{~kJ} / \mathrm{mol}\left(R^{2}=0.999\right)$ for the BPO initiator and $94.9 \mathrm{~kJ} / \mathrm{mol}\left(R^{2}=0.999\right)$ for AMBN, which are consistent with the reported literature values (i.e. $96.6 \mathrm{~kJ} / \mathrm{mol}$ for the BPO-initiated styrene polymerization) [30] and with the difference of the activation energies of the two initiators (i.e., about 124 and $128 \mathrm{~kJ} / \mathrm{mol}$ for the thermal decomposition of BPO and AMBN, respectively) [15, 31].

As can be seen, the addition of GTR results in a decrease in the $E_{\text {eff }}$ value for the BPOinitiated polymerization. This can be attributed to the strong interactions between the peroxide 


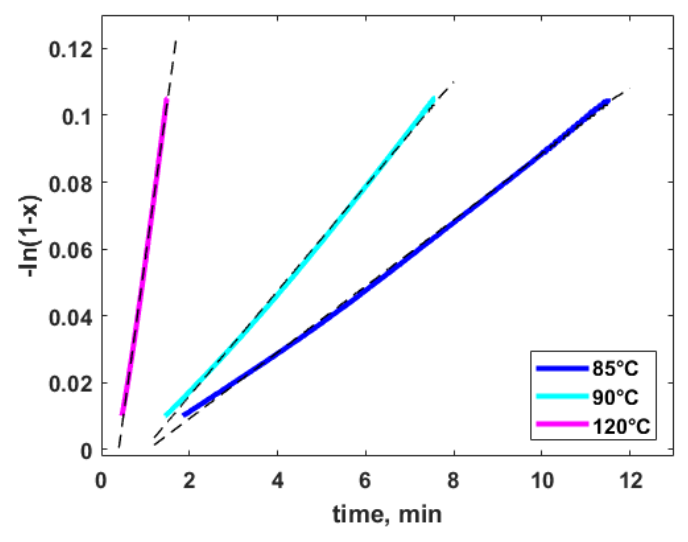

(a)

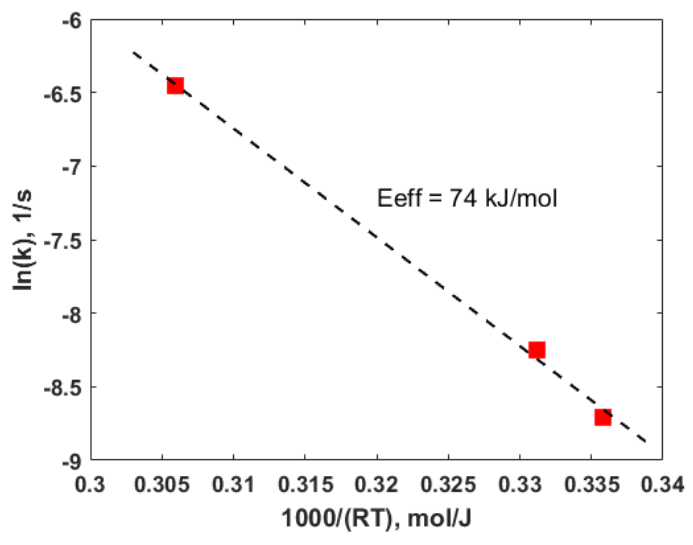

(b)

Figure 7: Calculation of the a) overall kinetic constant $k$ and b) effective activation energy $E_{\text {eff }}$ for the BPO-initiated styrene polymerization, in the presence of GTR (30 wt\%).

initiator and the surface of carbon black that, as described earlier, may induce a redox initiation, thus accelerating the decomposition of BPO. According to Eq. (2), the effective activation energy of the polymerization will be given by the expression: $E_{\text {eff }}=E_{p}+0.5^{*}\left(E_{d^{-}} E_{t}\right)$, where the subscripts $p, d$ and $t$ correspond to the propagation, decomposition and termination reactions, respectively. As a result, a decrease of the value of $E_{d}$ within the range defined by the activation energy values of redox initiation (i.e. $40-60 \mathrm{~kJ} / \mathrm{mol}$ ) and thermal homolysis (i.e., around 124 and $128 \mathrm{~kJ} / \mathrm{mol}$ for BPO and AMBN, respectively) [15], would result in a subsequent decrease of the overall value of $E_{\text {eff }}$. It should be noted that at this point that, other parallel interactions are expected to take place (e.g., inhibition and/or retardation by the additives of GTR) that might have a positive or negative influence on the value of the effective activation energy. Hence, the above explanation could only account for a part of the observed overall decrease. Nonetheless, since the interactions between BPO and the surface of carbon black are extensive in this system $[17,18]$, it is expected that their role will be dominant in the observed decrease of the value of $E_{\text {eff }}$.

On the other hand, the effect seems to be reversed in the case of AMBN, where an increase is observed on the calculated values of $E_{\text {eff }}$ upon addition of GTR. In this case, in the absence of strong interactions between the initiator and carbon black, the observed behavior can be mainly attributed to the overall inhibition effect of GTR on the course of the polymerization. This inhibition, also demonstrated in Figure 3, is primarily caused by the various additives and impurities present in the GTR formulation. In the case of strong inhibition and for low monomer conversion (i.e., for negligible initiator and inhibitor(s) concentrations), the effective activation energy will be calculated by an expression of the type: $E_{e f f}=E_{p}+E_{d^{-}} E_{z}$, where $E_{z}$ corresponds to the overall activation energy of the inhibition reactions. It is certainly difficult to assess the exact contribution of the inhibition activation energy on the overall value of $E_{\text {eff }}$, but its combination with the substantial increase induced by the decomposition term (i.e., due to the fact that $E_{d}$ is no longer multiplied by 0.5 ) seems to result in the overall increasing effect of the value of $E_{\text {eff }}$. Note that, given the assumptions involved in the method used to calculate the value of $E_{\text {eff }}$ and the complexity of the associated chemical reactions mechanism, it would be rather risky to extend this discussion further than the mentioned overall trends, on the basis of the exact calculated values.

\section{Conclusions}

In this investigation, the aim was to asses the reaction kinetics of the free-radical bulk polymerization of styrene in presence of fine rubber particles from recycled used tires, used as mechanical property modifier in stiffness polymers. For this purpose, the course of the reaction was monitored by DSC under different conditions in terms of temperature and composition, and two chemical initiators of different nature, BPO and AMBN. This research has shown that the addition of GTR 
Table 2: Calculated activation energy $E_{\text {eff }}$ values for styrene polymerization in absence and presence of GTR for different mass ratios of initiator (I) to styrene (St). Parenthesis are $R^{2}$ values of the linear regression.

\begin{tabular}{ccccc}
\hline GTR (wt\%) & $\begin{array}{c}\mathrm{I} / \mathrm{St} \\
(\mathrm{wt} / \mathrm{wt} \%)\end{array}$ & $\begin{array}{c}\mathrm{I} / \mathrm{GTR} \\
(\mathrm{wt} / \mathrm{wt} \%)\end{array}$ & $\begin{array}{c}E_{\text {eff }}(\mathrm{kJ} / \mathrm{mol}) \\
\text { for BPO }\end{array}$ & $\begin{array}{c}E_{\text {eff }}(\mathrm{kJ} / \mathrm{mol}) \\
\text { for AMBN }\end{array}$ \\
\hline 0 & 4.6 & & $92.7_{\left(R^{2}=0.999\right)}$ & $94.9_{(0.999)}$ \\
48 & 4.6 & 4.6 & $70.5_{\left(R^{2}=0.908\right)}$ & $140.3_{\left(R^{2}=0.998\right)}$ \\
48 & 9.4 & 9.4 & $76.1_{\left(R^{2}=0.991\right)}$ & $113.3_{\left(R^{2}=0.998\right)}$ \\
29 & 4.6 & 10.8 & $74.0_{\left(R^{2}=0.997\right)}$ & $111.8_{\left(R^{2}=0.999\right)}$ \\
29 & 6.6 & 15.5 & $45.6_{\left(R^{2}=0.863\right)}$ & $109.9_{\left(R^{2}=0.999\right)}$ \\
\hline
\end{tabular}

deviates the evolution of the polymerization and diminish the rate of reaction when using any of the two initiators. The strongest effects were observed for the BPO initiated system, which displays a severe retardation and inhibition that increase with the content of rubber particles and lead to low monomer conversions. In contrast, the reaction initiated with AMBN is moderately retarded and slightly inhibited only at high GTR percentages. These particular effects were mainly attributed to the chemical interactions between the initiator radicals of the carbon black, additives and impurities present in the formulation of the GTR. It was also shown that in the case of the $\mathrm{BPO}$, the effective activation energy decreases compared to the one required for the polymerization of the neat monomer, while the effect was reversed in the case of AMBN where the final activation energy increased.

The present study confirms previous findings and supports the idea that the mechanism of the BPO initiated free-radical polymerization of styrene with GTR is a complex system in which different physicochemical phenomena intervene and several and antagonistic reactions may take place simultaneously, making it difficult to elucidate them separately. Finally, additional research is being done to assess the general mechanism of the kinetics of the inhibition and determine the inhibition constant values for this system.

\section{References}

[1] S. Ramarad et al. "Waste tire rubber in polymer blends: A review on the evolution, properties and future". en. In: Progress in Materials Science 72 (July 2015), pp. 100-140.

[2] Dan Dobrota and Gabriela Dobrota. "An innovative method in the regeneration of waste rubber and the sustainable development". en. In: Journal of Cleaner Production 172 (Jan. 2018), pp. 3591-3599.

[3] James E Mark, Burak Erman, and F. R Eirich. Science and technology of rubber. English. 1994.

[4] D. Tuchman and S. L. Rosen. "The mechanical properties of plastics containing cryogenically ground tire". en. In: Journal of Elastomers 6 P Plastics 10.2 (Apr. 1978), pp. 115-128.

[5] J. Karger-Kocsis, L. Mészáros, and T. Bárány. "Ground tyre rubber (GTR) in thermoplastics, thermosets, and rubbers". en. In: Journal of Materials Science 48.1 (Jan. 2013), pp. 1-38.

[6] M. Pittolo and R. P. Burford. "Rubber-crumb modified polystyrene". In: Journal of materials science 21.5 (1986), pp. 1769-1774.

[7] A. Brydon, G. M. Burnett, and G. G. Cameron. "Free-radical grafting of monomers to polydienes. I. Effect of reaction conditions on grafting of styrene to polybutadiene". In: Journal of Polymer Science: Polymer Chemistry Edition 11.12 (Dec. 1973), pp. 3255-3269.

[8] Serena Coiai et al. "Modification of Cross-Linked Rubber Particles by Free Radical Polymerization". en. In: Macromolecular Symposia 234.1 (Feb. 2006), pp. 193-202.

[9] Dimitris S. Achilias and George P. Nikolaidis Alexandros K.and Karayannidis. "PMMA / organomodified montmorillonite nanocomposites prepared by in situ bulk polymerization". In: Journal of Thermal Analysis and Calorimetry 102.2 (Nov. 2010), pp. 451-460. 
[10] Dimitris S. Achilias. "Investigation of the radical polymerization kinetics using DSC and mechanistic or isoconversional methods". en. In: Journal of Thermal Analysis and Calorimetry 116.3 (June 2014), pp. 1379-1386.

[11] Ernesto L. Rodriguez. "The effect of free radical initiators and fillers on the cure of unsaturated polyester resins". en. In: Polymer Engineering and Science 31.14 (July 1991), pp. 10221028.

[12] Ruohua Xiong. "Studies in polymer composites based on carbon containing pulverized rubber materials". PhD thesis. Chicago: Illinois Institute of Technology, July 2010.

[13] Oskar Bera et al. "A new approach for the kinetic modeling of free radical bulk polymerization of styrene". en. In: Polymer Journal 43.10 (Oct. 2011), pp. 826-831.

[14] F. L. Marten and A. E. Hamielec. "High-conversion diffusion-controlled polymerization of styrene. I". In: Journal of Applied Polymer Science 27.2 (Feb. 1982), pp. 489-505.

[15] George G. Odian. Principles of polymerization. 4th ed. OCLC: ocm53393894. Hoboken, N.J: Wiley-Interscience, 2004.

[16] L. K. J. Tong and W. O. Kenyon. "Heats of Polymerization. III. Styrene and Substituted Styrenes". en. In: Journal of the American Chemical Society 69.6 (June 1947), pp. 1402-1405.

[17] Gerard Kraus, J. T. Gruver, and K. W. Rollmann. "Inhibition of polymerization by carbon blacks". In: Journal of Polymer Science 36.130 (Apr. 1959), pp. 564-565.

[18] K. Ohkita et al. "The free radical polymerization of vinyl monomers in the presence of carbon black". en. In: Carbon 13.5 (1975), pp. 443-448.

[19] Suda Kiatkamjornwong and Pisnu Pomsanam. "Synthesis and characterization of styrenicbased polymerized toner and its composite for electrophotographic printing". en. In: Journal of Applied Polymer Science 89.1 (July 2003), pp. 238-248.

[20] Kazuhiro Fujiki, Norio Tsubokawa, and Yasuo Sone. "Radical Grafting from Carbon Black. Graft Polymerization of Vinyl Monomers Initiated by Azo Groups Introduced onto Carbon Black Surface". In: Polymer Journal 22.8 (Aug. 1990), pp. 661-670.

[21] Ljubisa R. Radovic. "Chemistry and Physics of Carbon, Vol. 27 Ljubisa R. Radovic, Ed. Marcel Dekker, Inc.: New York, 2001. 416 pp +XV. ISBN 0-8247-0246-8. \$225". en. In: Energy \&f Fuels 15.2 (Mar. 2001), pp. 502-502.

[22] Norio Tsubokawa, Kazuhiro Fujiki, and Yasuo Sone. "Radical Grafting from Carbon Black. Graft Polymerization of Vinyl Monomers Initiated by Peroxyester Groups Introduced onto Carbon Black Surface”. In: Polymer Journal 20.3 (Mar. 1988), pp. 213-220.

[23] Kumakazu Ohkita, Norio Tsubokawa, and Eiichi Saitoh. "The competitive reactions of initiator fragments and growing polymer chains against the surface of carbon black". en. In: Carbon 16.1 (1978), pp. 41-45.

[24] Turner Alfrey and Charles C. Price. "Relative reactivities in vinyl copolymerization". In: Journal of Polymer Science 2.1 (Feb. 1947), pp. 101-106.

[25] S. T. Balke and A. E. Hamielec. "Bulk polymerization of methyl methacrylate". In: Journal of Applied Polymer Science 17.3 (Mar. 1973), pp. 905-949.

[26] Kumakazu Ohkita et al. "Influence of oxygen-containing groups of carbon black on the thermal polymerization of styrene". en. In: Nippon Gomu Kyokaishi 49.12 (1976), pp. 900-907.

[27] Peter A. Ciullo and Norman Hewitt. Compounding materials. Elsevier, 1999, pp. 4-49.

[28] Nicolas Sbirrazzuoli and Sergey Vyazovkin. "Learning about epoxy cure mechanisms from isoconversional analysis of DSC data". en. In: Thermochimica Acta 388.1-2 (June 2002), pp. 289-298.

[29] V.N. Kondratiev. "Chain Reactions". en. In: Comprehensive Chemical Kinetics. Vol. 2. Elsevier, 1969, pp. 81-188.

[30] Paul J. Flory. Principles of polymer chemistry. eng. 19. print. Ithaca, NY: Cornell Univ. Press, 2006.

[31] "Initiators and Reactor Additives for Thermoplastics". In: (Jan. 2013). Posted at http:// www.akzonobel.com. 